\title{
Características de los talleres extraescolares de los establecimientos educacionales de la Araucanía, Chile \\ Characteristics of extracurricular activities of educational establishments in the Araucanía, Chile
}

\author{
Noemí Salvador Soler, Andrés Cristian Uribe Delgado, Carlos Roberto Arriagada Hernández \\ Universidad Autónoma de Chile (Chile)
}

\begin{abstract}
Resumen. Los talleres extraescolares favorecen al desarrollo integral de los estudiantes mediante el estímulo del desarrollo de diversas habilidades, capacidades y/o talentos. Además, representan una oportunidad para contrarrestar los niveles de sedentarismo que presentan los y las jóvenes a nivel escolar. Objetivo: Determinar las características de los talleres extraescolares de los establecimientos educacionales de la Araucanía (Chile). Metodología: La muestra incluyó a 48 responsables de taller pertenecientes a 11 colegios de dependencia municipal o particular subvencionada de la región. Para la evaluación de las características de los talleres implementados en los distintos establecimientos se aplicó un cuestionario que contempla tres dimensiones: características generales, recursos materiales y experiencia de los docentes encargados de cada taller. Resultados: Los talleres evaluados son mayormente de categoría deportiva, de orientación formativa e impartidos por docentes con más de tres años de experiencia, principalmente profesores de Educación Física, varones y con una edad entre 31 y 40 años. Respecto a los materiales y espacios, si bien los talleres cuentan con una cantidad suficiente de materiales principalmente de buena calidad, más de la mitad de talleres se desarrollan en espacios como cancha, patio o pasillo. Conclusión: El estudio pone en evidencia la escasez de talleres extraescolares de orientación recreativa, la falta de espacios aptos para el deporte, así como la poca representación femenina tanto a nivel de docentes o responsables de los talleres como de participantes de los mismos.
\end{abstract}

Palabras clave: taller deportivo, taller extraescolar, formación, aprendizaje activo, educación física, tiempo libre, educación y recreación.

Abstract. Extracurricular activities benefit the integral development of students by stimulating the development of various abilities, capacities and / or talents. In addition, they represent an opportunity to counteract the levels of sedentary lifestyle that young people present at the school level. Objective:To determine the characteristics of the extracurricular activities of the educational establishments of the Araucanía, Chile. Methodology:The sample included 48 responsables of the extracurricular activities from 11 municipal or private subsidized schools in the Araucanía region. For the evaluation of the characteristics of the extracurricular activities implemented in the different establishments, a questionnaire of three dimensions (general characteristics, material resources and experience of the responsable in charge) was applied. Results: The evaluated extracurricular activities are mostly of the sports category, of formative orientation and given by responsables in charge with more than three years of experience, mainly Physical Education teachers, men and with an age between 31 and 40 years. Regarding materials and spaces, the extracurricular activities have enough quantity of materials and the mainly good quality, but more than half of these activities take place in spaces such as the school court, schoolyard or hallway class. Conclusion:The study highlights the scarcity of recreational orientation activities, the lack of spaces suitable for sports, as well as the low representation of women both at the level of teachers and of participants.

Keywords: sports activities, extracurricular activities, training, active learning, physical education, after-school time, education and recreation.

\section{Introducción}

De acuerdo con el Decreto $\mathrm{N}^{\circ} 290$, la educación en Chile contempla desde 1984 las actividades extracurriculares, ya que éstas contribuyen a buscar en la educación un desarrollo integral de niños y jóvenes (Decreto $\mathrm{N}^{\circ} 290$, 1984). El decreto $\mathrm{N}^{\circ} 290$ se encuentra vigente hasta el día de hoy, y es el que crea y organiza el Departamento de Educación Extraescolar, el cual es dependiente de la Secretaría y Administración General del Ministerio de Educación (MINEDUC), teniendo a su cargo acciones educativas en horarios no lectivos de los establecimientos. La definición según el Decreto

Fecha recepción: 14-05-21. Fecha de aceptación: 19-10-21

Noemi Salvador Soler

noemi.salvador@uautonoma.cl
$\mathrm{N}^{\circ} 290$ (1984) de Educación Extraescolar obedece al:

Conjunto de acciones educativo-recreativas de tiempo libre que se origina por la práctica orientada y organizada de actividades deportivas, artísticas, científicas, cívico-sociales y en general de todas aquellas que, en función de los fines y objetivos de la educación nacional, contribuyan al desarrollo de la persona. (p.1)

Bahamonde, Carmona, Albornoz, Hernández-García y Torres-Luque (2019) afirman que este tipo de actividades físicas extraescolares benefician a nivel físico obteniendo mejoras significativas en parámetros relacionados con actividades que involucren fuerza o velocidad. Sumado al beneficio y mejora sustancial de la calidad de vida y disminución del sedentarismo (Sanabria, Silveira, Guillen \& Pérez, 2019). Con ello los estudiantes podrán desarrollar sus capacidades, habilidades y actitudes en el contexto escolar, brindando mayores opor- 
tunidades de relación fuera del aula de clases, que permitan desplegar una mejor convivencia escolar. Además, estas actividades extracurriculares que se realizan en las instituciones educativas de enseñanza básica, media o superior favorecen la utilización del tiempo libre generando un apoyo para los padres y apoderados que durante ese periodo de tiempo realizan actividades domésticas o laborales (Nogueira, 2008).

Si bien las actividades extracurriculares descritas anteriormente fueron acuñadas inicialmente por el término «Educación Extraescolar», este concepto contiene una doble significación. Por una parte, el prefijo «Extra» podría significar »fuera de» haciendo referencia a tiempo y a espacio, en este caso, la escuela. Por otra parte, también podría significar «además de» haciendo referencia a tiempo y a un contenido o disciplina a tratar. De acuerdo con las características de la educación extraescolar chilena, entendida como un elemento que forma parte del proceso formativo de la escuela fuera del horario escolar compuestas por actividades complementarias (actividades artísticas y deportivas), con participación voluntaria u obligatoria dependiendo de las características del taller y de la organización escolar del establecimiento (Calero, 2016). Por otro lado, ante las dificultades semánticas del término, finalmente pasaron a llamarse actividades curriculares no lectivas o talleres de Jornada Escolar Completa (en adelante, JEC) en el caso de los establecimientos educativos subvencionados por el Estado dentro del plan de Jornada Escolar Completa (Millalén, 2008). No obstante, en el caso de los establecimientos privados estas actividades suelen ser denominadas actividades extraprogramáticas o actividades de libre elección. Por lo que para el presente trabajo de investigación se mantendrá el término inicial de actividades o talleres extraescolares, considerando que serán aquellas actividades que sean desarrolladas como un complemento al currículum y de carácter no lectivo.

En relación con el uso del tiempo libre en la etapa escolar, según Millalén (2008) dentro las características principales que llevó al MINEDUC a implementar la JEC es el tiempo adicional que estarían los alumnos y alumnas en los establecimientos. De acuerdo con el MINEDUC, un mayor tiempo en el colegio podría ayudar a profundizar el proceso de aprendizaje intelectual y moral, lo que conlleva a examinar el currículo educacional en las modalidades de enseñanza y evaluación al igual que la regulación de las normas y las actividades que se hacen fuera del horario escolar. También el tiempo destinado a actividades extraescolar deportivas, forma- tivas y recreativas contribuyen a la prevención de la violencia escolar, siendo la práctica deportiva una herramienta de aprendizaje de actitudes y conductas adecuadas (Medina \& Reverte, 2019). Por otra parte, Salazar (2020) evidencia mediante un modelo de taller deportivo, denominado programa de fútbol formativo extraescolar, una mejora de la condición motriz en estudiantes adolescentes, lo que favorece a su desarrollo integral. Es en este contexto que desde el año 1997 se ha implementado la ley 19532/1997, la cual crea el régimen de la JEC y dicta normas para su aplicación. Además, dicha ley tiene como objetivos mejorar la infraestructura de los establecimientos, así como también la calidad de la educación aumentando las horas de clases. Además, la ley 19532 promueve la educación valórica, la igualdad de oportunidades para todas las clases sociales para construir y fortalecer la democracia en todos los espacios de la vida ciudadana (García-Huidobro \& Concha, 2009). En Chile, la reforma educacional de aquella época que incluyó la JEC fue descrita como un proceso gradual con el objetivo básico de mejorar la calidad de los aprendizajes y aumentar la equidad en su distribución social mediante un cambio por ende de los objetivos planteados en el Marco Curricular (DESUC, 2005). La Ley que introdujo la JEC permitiría aumentar en un 30\% el tiempo de permanencia de los estudiantes de $3^{\circ}$ básico a $4^{\circ}$ medio en los establecimientos que recibieran aportes del Estado como eran los colegios particulares subvencionados o municipales (Calvo, 2013).

Según Martinic (2015) el tiempo de extensión de la jornada escolar contribuye positivamente en el aprendizaje de los estudiantes, pero este depende en gran medida del uso que se haga con ese tiempo, cuando mayor parte del tiempo se utilice en tareas que no fomentan el desarrollo integral del estudiante este tiempo extra no producirá ganancia alguna. En España el tiempo de ocio y su práctica tiene que ver más con motivaciones personales de los estudiantes que actividades organizadas por los centros (Ponce de León, Sanza, Sáenz \& Alonso, 2019). Por otra parte, un estudio mexicano concluye que las actividades en el tiempo no lectivo permiten que los estudiantes refuercen otro tipo de conocimientos cuando existen actividades planificadas, reforzando de esta manera el aprendizaje de los estudiantes (Gurrola, Pimienta \& Sil, 2015). En Brasil, Distrito Federal, el tiempo es una categoría social fundamental para el desarrollo de capital cultural del alumnado, pero este tiempo no se utiliza eficientemente e impacta en sobrecarga, lo que quita tiempo social fue- 
ra de la escuela (Cruz \& Castioni, 2017). En síntesis, el tiempo es un factor clave para la construcción de un currículum escolar centrado en el desarrollo integral del estudiante y para el progreso de la calidad educativa.

En este sentido, en Chile la JEC contribuiría al desarrollo integral de todos los estudiantes, ya que las escuelas son una institución compleja donde se busca la formación y crecimiento de los alumnos y este constituye el espacio social principal para el desarrollo integral de los estudiantes, a través de implementar actividades escolares que desarrollen valores en los estudiantes, al responder a sus necesidades pedagógicas y sociales (DESUC, 2005).

En Chile, el desarrollo integral del estudiante se entiende por la promoción de conocimientos, habilidades y actitudes que les permitan desarrollar autonomía, apreciar sus características personales, desarrollar su capacidad motora, valorar el cuidado del cuerpo, desarrollar actitudes de respeto y aceptación de la diversidad social, étnica, cultural, religiosa y física, desarrollar la curiosidad y la creatividad, expresarse libre y creativamente a través de diferentes lenguajes artísticos (Ley $\left.N^{\circ} 20370,2009\right)$. De acuerdo con Millalén (2008) para garantizar el desarrollo integral del estudiante, en Chile los talleres extraescolares y/o talleres JEC deberían estar a cargo de profesores o monitores capacitados en la aplicación de nuevas metodologías que beneficien el aprendizaje integral dentro de una actividad pedagógica y contextualizarse en relación con las características de los alumnos y sus familias. Además, Calero (2016) afirma lo siguiente:

En estas actividades juega un factor de peso para la ocurrencia de experiencias óptimas que los estudiantes participan por un interés personal. También se considera importante, que las actividades deportivas y artísticas poseen características que permiten el control sobre el devenir de las actividades y la retroalimentación sobre el propio desempeño facilitada por las reglas claras con cierto margen de decisión. (p. 108)

En este mismo sentido, para Calvo (2013) los profesores deberían hacer un uso efectivo del tiempo durante las clases o talleres JEC, ya que la calidad de las instrucciones irá relacionada con el aprendizaje de los alumnos. Para el logro de dichos fines se debe contar con profesores preparados para lograr una implementación eficiente de los talleres (Carreño, Díaz, López \& Martín, 2019). Parra, Oliva y Suárez (2009) señalan que para que un programa extracurricular sea efectivo para el logro de los aprendizajes debe tener ciertas caracte- rísticas como realizarse en un lugar estable y seguro, también debe tener una estructura clara en la cual los estudiantes sientan que estos talleres tienen límites de convivencia. Otra característica importante de los programas que los mencionados autores señalan y contribuye a su éxito es que los talleres aporten experiencias relevantes y desafiantes para los estudiantes, siendo estos un aporte para la promoción de la actividad física en contextos escolares (Marambio et al., 2020).

Como se ha mencionado anteriormente son diversos los autores que señalan la importancia de las instalaciones o las capacitaciones y planificaciones pedagógicas de los docentes encargados. Sin embargo, los talleres también deberían cumplir con otras características relacionadas con los recursos y/o horarios, entre otros. Por lo que la pregunta de investigación sería ¿cuáles son las características de los talleres extraescolares que se están desarrollando actualmente y su aporte al desarrollo integral de los estudiantes?

\section{Material y métodos}

La investigación fue de enfoque cuantitativo, tipo no experimental, corte transversal y alcance descriptivo. La muestra fue seleccionada bajo un criterio no probabilístico y por conveniencia, la cual incluyó a 48 responsables de taller pertenecientes a 11 colegios, con una matrícula promedio de 661 estudiantes por establecimiento y de dependencia municipal o particular subvencionada de la región de la Araucanía (Chile). Los responsables de taller fueron contactados vía email y RRSS facilitándoles un enlace digital de acceso al cuestionario a fin de que aquellos que mostrasen interés en la investigación pudiesen completar anónimamente dicho cuestionario. Como criterios de inclusión fueron considerados todos aquellos responsables de taller que aceptaron participar en el estudio enviando de forma voluntaria el formulario online con el cuestionario (Google Forms) y que firmaron el consentimiento informado que autoriza el uso de la información con fines científicos. No fue aplicado ningún criterio de exclusión ya que todas las preguntas eran de tipo cerrado, de respuesta obligatoria y además no se observó ningún tipo de respuestas que en su conjunto mostrasen algún tipo de incoherencia interna. Para la recogida de información se diseñó un cuestionario el cual fue validado por un grupo de expertos. El cuestionario está formado por tres dimensiones: una sobre las características generales de los talleres (denominación, categoría y orientación), otra sobre los recursos materiales (calidad y 
cantidad de los materiales o implementos) y una tercera sobre características sociodemográficas y de experiencia laboral de los responsables de taller. El estudio fue desarrollado siguiendo lo expuesto en la Declaración de Helsinki respecto a la confidencialidad de los datos y la obtención de un consentimiento informado por parte de los participantes.

\section{Análisis estadístico}

Tras la recopilación de datos estos fueron descargados en Excel y posteriormente importados y analizados con el software SPSS versión 22.0. En cuanto a la estadística descriptiva aplicada se calcularon frecuencias y porcentajes de cada una de las preguntas del cuestionario. No se pudieron calcular los resultados de las pruebas estadísticas de independencia entre variables categóricas que se realizaron junto con las tablas cruzadas, ya que al aplicarse fueron obtenidos valores inferiores a 5 en más de un 20\% (SPSS, 2021).

\section{Resultados}

En relación con la denominación de los talleres y las categorías de los 48 talleres implementados en las horas de libre disposición en los 11 establecimientos educacionales que forman parte de los planes de estudio, la mayoría eran extraescolares (27), seguidos por la denominación taller deportivo (15) y en una minoría (6) están los denominados extraprogramáticos. Por otra parte, en cuanto a las categorías predominan claramente las categorías deportivas (37) y artísticas (11) en segundo lugar, no existe ningún taller de reforzamiento o relacionado con el aumento de horas de una asignatura. Además, la tabla cruzada permite observar que la mayoría de los talleres extraescolares son de categoría deportiva (Tabla 1).

\begin{tabular}{|c|c|c|c|c|}
\hline \multicolumn{3}{|c|}{$\begin{array}{l}\text { Tabla } 1 \\
\text { Tabla cruzada sobre la denominación del taller y la categoría }\end{array}$} & Categoría & \multirow{3}{*}{$\begin{array}{c}\text { Total } \\
6\end{array}$} \\
\hline \multicolumn{2}{|c|}{ Denominación } & Artístico & Deportivo & \\
\hline Extraprogramático & Frecuencia & 4 & 2 & \\
\hline & $\%$ & 36.4 & 5.4 & 12.5 \\
\hline \multirow[t]{2}{*}{ Extraescolar } & Frecuencia & 7 & 20 & 27 \\
\hline & $\%$ & 63.6 & 54.1 & 56.3 \\
\hline \multirow[t]{2}{*}{ Taller deportivo } & Frecuencia & 0 & 15 & 15 \\
\hline & $\%$ & 0 & 40.5 & 31.3 \\
\hline Total & & 11 & 37 & 48 \\
\hline
\end{tabular}

Al observar y relacionar las frecuencias con la orientación y categoría, se puede observar que la mayoría de los talleres son descritos como formativos y la minoría como competitivos. Además, la orientación formativa fue predominante también dentro de los mismos talleres deportivos (Fig. 1). Es de destacar por tanto que dentro de los talleres de categoría deportiva la orientación competitiva es la menormente representada. En relación con los talleres artísticos, éstos son recreativos o formativos en partes casi iguales.

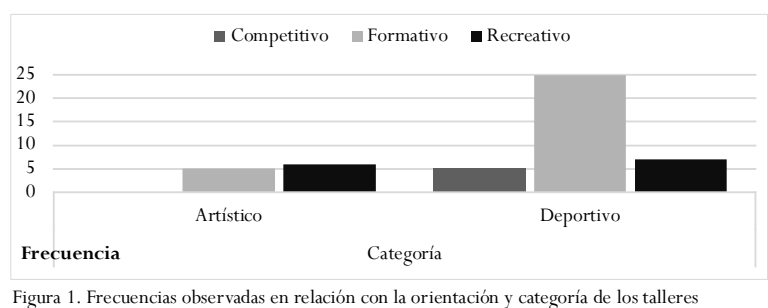

En relación con el cupo y género de los 48 talleres implementados en las horas de libre disposición la mayoría son mixtos (23), seguidos por los dirigidos al género masculino (16) y en una minoría (9) al género femenino. Respecto al cupo son mayoritariamente para más de 20 estudiantes. Al cruzar ambas variables se puede observar que los talleres mixtos son los predominantes para los cupos de más de 20 y también entre 10 y 20 (Tabla 2).

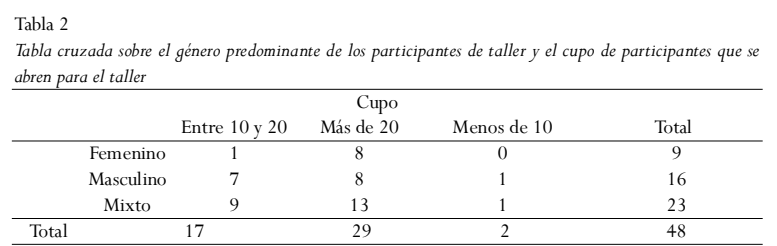

Se aprecia que los establecimientos educacionales emplean como espacio para los talleres principalmente el gimnasio (16), seguido por la utilización del pasillo (10) y en tercer lugar las canchas (8). Mientras que los espacios menos utilizados para dichos talleres serían la multicancha, el patio o la sala entre otros (Fig. 2).

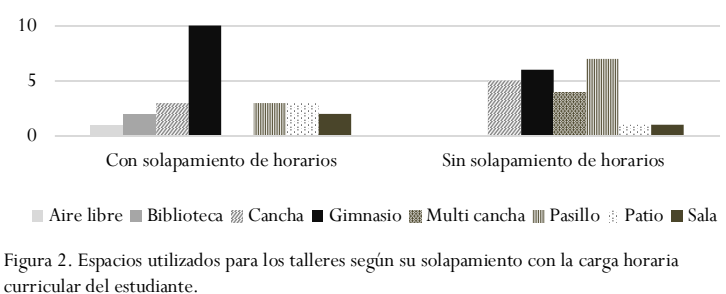

Respecto al horario del taller y su solapamiento con la carga horaria curricular de los estudiantes, en la Figura 3 se puede observar que la mitad de ellos se imparten durante la carga horaria curricular del estudiante (24), siendo además la mayoría de ellos denominados extraescolares (12).

En relación con la denominación de los talleres, la Figura 3 muestra que, si bien la mayoría son denominados extraescolares, después de esta denominación pre- 


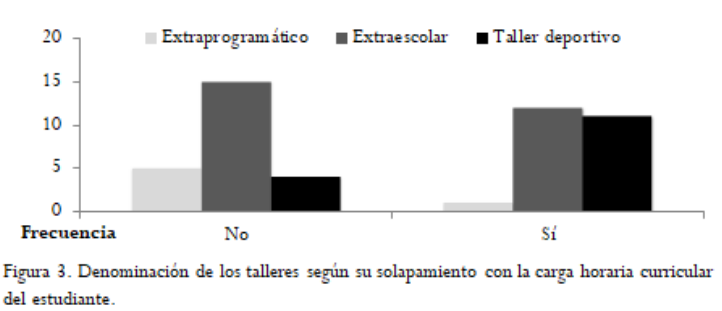

domina la denominación de taller deportivo en el ámbito extraescolar. Además, la mayor parte de los talleres deportivos son implementados en las horas de libre disposición que permiten actualmente las bases curriculares.

En cuanto a los materiales, los resultados muestran que la mayoría son de buena calidad y hay suficientes (91.3\%), es decir no sobran (Tabla 3). Este fenómeno podría deberse a la existencia de nuevas subvenciones que permiten a los establecimientos adquirir los materiales necesarios para la ejecución de los talleres extraescolares.

Tabla 3
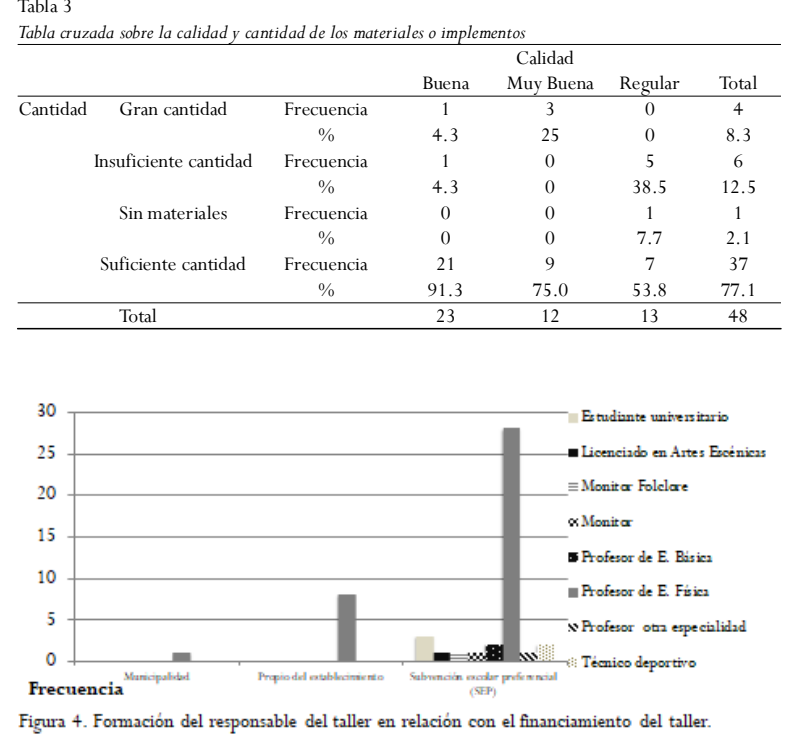

En relación con los responsables de taller, la Figura 4 muestra que, si bien existe una amplia variedad de tipos de responsables de taller en relación con su formación, la gran mayoría de estos son Profesores de Educación Física. Además, la mayor parte de los talleres son financiados mediante la subvención escolar preferencial (SEP).

En relación con la edad, la Tabla 4 muestra que el $50 \%$ de los responsables de taller tienen entre $31-40$

Tabla 4

\begin{tabular}{ccccccc}
\multicolumn{6}{l}{ Tabla cruzada en relación con la edad y experiencia de los responsables de taller } \\
\begin{tabular}{ccccccc}
\multicolumn{5}{c}{ Experiencia (años) } \\
& & & $\leq 1$ & Entre $1-3$ & $>3$ & Total \\
\hline Rango etario & $20-30$ & Frecuencia & 1 & 3 & 9 & 13 \\
(años) & & $\%$ & 100 & 100 & 20.5 & 27.1 \\
& \multirow{2}{*}{$31-40$} & Frecuencia & 0 & 0 & 24 & 24 \\
& & $\%$ & 0 & 0 & 54.5 & 50 \\
& $>41$ & Frecuencia & 0 & 0 & 11 & 11 \\
& & $\%$ & 0 & 0 & 25 & 22.9 \\
\hline Total & & & 1 & 3 & 44 & 48 \\
\hline
\end{tabular}
\end{tabular}

años y el otro 50\% corresponderían a responsables de entre 20-30 años (27.1\%) y >41 años (22.9\%). En cuanto a la experiencia en el taller, la gran mayoría tienen más de 3 años de experiencia.

Respecto del género, predominan mayoritariamente los responsables encargados de taller de género masculino $(75 \%)$, mientras que aquellos encargados de género femenino solo un representaron un 25\% del total (Fig. 5).

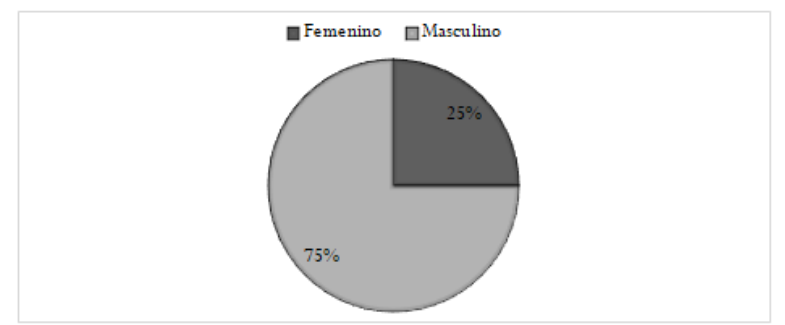

Figura 5. Genero del responsable del taller.

\section{Discusión}

En relación con las características generales de los 48 talleres implementados, los resultados muestran una clara predominancia de los talleres de categoría deportiva, lo que ofrece una buena expectativa de desarrollo para la carrera de Pedagogía en Educación Física. Por otra parte, la mayor oferta de talleres extraescolares de carácter deportivo ofrece mayores oportunidades para contrarrestar los niveles de sedentarismo presentes en menores de 5 a 17 años, que alcanzan un 48.4\% en Chile (MINDEP, 2019). Hermoso y Chinchilla (2010) afirman que los escolares presentan valores altos en la satisfacción en la ocupación de su tiempo libre. En este sentido la población escolar y su inclinación por la participación en talleres extraescolares deportivos favorecerían, a generar una interacción positiva con la actividad física y directamente relacionado, a un aumento de los componentes favorecedores de salud (Rosa, García \& Carrillo, 2019).

En cuanto a la variedad de talleres no se encontró ninguno de categoría cognitiva lo que podría explicarse principalmente porque preferentemente esas horas de libre disposición son asignadas para profesores de educación física y monitores (talleres artísticos, deportivos u otros). Además, en este sentido los establecimientos realizan algún grado de promoción social del deporte (Sandoval \& García, 2014), lo que beneficia en la participación de toda la comunidad escolar. Si consideramos los planteamientos de la JEC en su denominación de formación integral, es complementario a la formación cognitiva la formación fisiológica, como la práctica de 
algún deporte, expresión artística o actividad física y esto podría explicar su abundante presencia (Ley $\mathrm{N}^{\circ}$ 19532, 1997).

La orientación que reciben los talleres extraescolares hace alusión a los objetivos que son asignados a cada uno, por lo que aquí aparece un punto importante de discusión ya que según el MINEDUC (2013) estos talleres extraescolares deben orientarse hacia la integralidad del proceso educativo, debe complementar el proceso cognitivo, considerando siempre los procesos cognitivos y actitudinales que atraviesan el currículum escolar chileno. De acuerdo con el Instituto Nacional de Deportes (IND, 2020), las orientaciones «recreativo», «formativo» $\mathrm{y}$ «competitivo» se encuentran definidas $\mathrm{y}$, al respecto se señala:

Se entiende por Deporte Recreativo al conjunto de actividades físicas efectuadas en el tiempo libre, practicadas según reglas de las especialidades deportivas o establecidas de común acuerdo por los participantes, con el fin de propender a mejorar la calidad de vida y la salud de la población, así como fomentar la convivencia familiar y social (p. 37).

Según los responsables de los talleres, no más de 10 talleres se consideran en la categoría recreativa, lo cual es contrario a lo planteado por el MINEDUC y el IND quienes fomentan la realización del deporte recreativo, por tanto, se relaciona de manera incipiente con el espíritu de la JEC que pretende desarrollar integralmente a los escolares en actividades activas y recreativas, esto se debe a que mayoritariamente están involucrados en actividades lectivas cognitivas y sedentarias. Por otra parte, si consideramos la orientación «formativa» de los talleres extraescolares participantes el IND (2020) señala:

Se entiende por formación para el deporte la puesta en práctica en niños, niñas, jóvenes y adultos de procesos de enseñanza y aprendizaje a cargo de profesionales o técnicos especializados vinculados a la actividad físicadeportiva, cuyo objetivo es el desarrollo de aptitudes, habilidades y destrezas necesarias para la práctica de la actividad física y los distintos deportes; el conocimiento de los fundamentos éticos, técnicos y reglamentarios de las especialidades deportivas, y la práctica sistemática y permanente de actividades físico-deportivas (p. 25).

Según este estudio la gran mayoría de los talleres se autodenomina «formativo». Pero este planteamiento no se cumple, principalmente si hablamos de sistematización, periodicidad y profundidad de los contenidos tratados en los mismos. En cambio, esto se podría observar si los talleres deportivos tuvieran presencia en cada uno de los niveles educativos, por ejemplo: deporte específico de tercero y cuarto año de educación general básica, el mismo deporte específico en otro taller de quinto y sexto, y secuencialmente, otro taller del mismo deporte específico en séptimo y octavo año de educación básica. Esta simple denominación, distribución, consideración y secuencia hablaría claramente de un taller extraescolar deportivo formativo, pero en general en la mayoría de los talleres extraescolares, los participantes no se mantienen o no se secuencian, perdiendo así su calidad de formativo. Para que un taller sea considerado de calidad formativa según Acosta (2012) debe tener como finalidad contribuir al desarrollo integral del individuo abarcando procesos de iniciación y perfeccionamiento deportivo que tienen lugar en instituciones deportivas, gubernamentales y educativas como es el caso de este trabajo.

Por otra parte, la denominación taller extraescolar deportivo competitivo es la que más llama la atención, ya que todos los integrantes de la Educación Física saben que para competir hay que dedicar una gran cantidad de tiempo en la práctica y en la enseñanza, ya sea de táctica como técnica. Además, el IND (2020) señala: «el Deporte de Competición corresponde a las prácticas sistemáticas de especialidades deportivas, sujetas a normas y con programación y calendarios de competencias y eventos» (p. 51). Claramente, sería conveniente revisar que aquellos talleres que tienen la orientación competitiva realmente cumplan con las características enunciadas por el IND en relación con la dedicación y programas de competición. Esto abre un debate en la orientación de los talleres extraescolares, ya sea de las instituciones como de los responsables de los mismos. En resumen, la mayoría de ellos debería considerar la orientación recreativa y los que se consideren en las orientaciones formativa y competitivas deberían cumplir con algunos de los mencionados para considerarse como tal.

Según Baeza, Morales y Suazo (2017) las actividades artísticas (danzas, artesanías, etc.) y deportivas son las que poseen la mayor adherencia, siempre orientados a la recreación, ya que en los colegios predominan los deportes y existe un amplio porcentaje formativo. Los resultados del presente trabajo si bien están de acuerdo con los resultados de los autores mencionados en cuanto a la predominancia de talleres deportivos en cambio contrastan con la orientación que se les da, al ser éstos mayoritariamente de carácter formativo.

De acuerdo con MINDEP (2019) al comparar género masculino y femenino, las mujeres alcanzan un $52.7 \%$ y los varones un $44.3 \%$ entre los menores de 5 a 17 
años inactivos. Los resultados del presente estudio en relación con el cupo y género de los talleres extraescolares evaluados son coherentes con los resultados mencionados por el MINDEP ya que solamente una minoría de ellos fueron impartidos especialmente para el género femenino, lo que evidencia una baja oferta escolar para practicar actividad física o deportes enfocado a las damas de los distintos grupos etarios. Además, si se tiene en cuenta que la mayor cantidad de población del país son mujeres (51.1\%) (INE, 2017), estos resultados adquieren mayor relevancia por lo que se debería considerar una mayor presencia de talleres para mujeres en cada una de las instituciones educativas y con una oferta superior a las tendencias de actividad física femenina como son las danzas y las expresiones artísticas, sumadas a los deportes individuales y colectivos con práctica exclusivamente femenina por sobre la participación mixta, que también aparece mencionada por los participantes. En este sentido Beltrán, Sierra, Jiménez, González-Cutre, Martínez y Cervelló (2017) manifiestan que las actividades escolares en recreos y talleres extraescolares deberían dinamizarse, para que fuesen más activos y se ajusten de mejor manera a las preferencias de las participantes, las cuales, según Leyton, García, Fuentes y Jiménez (2018) muestran mayores necesidades psicológicas de relaciones sociales en el ejercicio que los hombres. Esto no apunta en ningún caso a disminuir los talleres masculinos para equiparar los talleres extraescolares, por el contrario, invita a mantener y elevar la oferta de práctica deportiva y de actividad física en las instituciones educativas.

Respecto al cupo de los talleres extraescolares evaluados, es interesante resaltar que al considerar el promedio de matrícula de 661 estudiantes de las instituciones educativas participantes (MIME.MINEDUC, 2021) y el promedio total de cupos de sus talleres extraescolares en su conjunto abarcaría aproximadamente un 10\% de atención de la población escolar por lo que difícilmente se podría conseguir un avance significativo en salud y educación integral.

En cuanto al solapamiento entre la carga horaria curricular de estudiante y los talleres extraescolares, es importante destacar que algunos responsables de taller señalaron que existen talleres extraescolares dentro de la jornada curricular en horario lectivo, lo que mostraría una incoherencia respecto al concepto de extraescolar, ya que tanto la doble jornada o la jornada escolar completa puede tener talleres extraescolares, siempre y cuando sea fuera del horario de la carga curricular. Según Arriagada y Calzadilla (2019) este fe- nómeno de desconocimiento respecto de elementos presentes en el currículum chileno tales como bases curriculares, planes de estudio, horas de libre disposición, entre otros, es frecuente en el profesorado y en cambio es de conocimiento y dominio de los equipos técnicos y directivos. Si bien ello no impacta de manera directa en la formación de los estudiantes, sí en los profesores, lo que es visualizado como un problema latente y actual.

Con respecto al espacio de realización se considera que un taller extraescolar deportivo o de cualquier índole o instancia educativa, necesita de algunos espacios básicos, mínimos e ideales para instalarse, funcionar y mantener la participación, aprendizaje y trascendencia en el tiempo (Bravo, 2015). En este sentido, los resultados de la presente investigación señalan que la mayoría de los talleres extraescolares que no se solapan con la carga horaria se desarrollan en el gimnasio, que sería el espacio considerado como ideal para su funcionamiento y desarrollo. Sin embargo, llama la atención que más de la mitad de los talleres que se desarrollan en espacios como cancha, patio o pasillo. Desde la perspectiva de las habilidades profesionales de adaptación e innovación, evidente en los profesores de Educación Física, es muy discutible analizar los tipos de talleres que pueden desarrollarse en un pasillo o en el patio entendiendo que suponen un espacio de transito común de toda la comunidad escolar, de características físicas no aptas para practicar deporte y que por lo general no cuentan con la implementación necesaria de arcos, aros, redes, líneas $\mathrm{u}$ otros elementos absolutamente necesarios para la enseñanza de contenidos deportivos. Por ello para Trujillo (2014) es necesario que todos los espacios sean evaluados y planificados para garantizar un aprovechamiento de estos, por ello se debe tener una mirada crítica hacia los espacios para que podamos regular y corregir de modo sistemático los procesos educativos generando a través de un análisis de los espacios interiores y exteriores que se puedan utilizar en la práctica diaria.

En relación a los recursos materiales con que funcionan los talleres extraescolares Manrique y Gallego (2013) señalan:

Para dar luces a esta respuesta, se realizaron rastreos bibliográficos de antecedentes locales que permitieron establecer que el uso del material didáctico de manera individual le proporciona al estudiante un aprendizaje exitoso, dado que la manipulación de dicho material fortalece la concentración y propicia aprendizajes más profundos, aspecto que invita a los docentes de hoy a renovar sus prácticas y las maneras de efectuarlas en el aula 
de clase (p. 8).

Dichas autoras destacan la importancia de la cantidad y diversidad de implementos o materiales deportivos para el uso individual, así como la calidad de los mismos, considerando que cumplan su función, es decir que den bote, que se equilibren, que no hagan daño a los usuarios, entre otros. Los resultados de este estudio son auspiciosos para el desarrollo de un taller extraescolar, ya que se menciona que todos ellos alcanzan en cantidad y son de buena calidad para que los niños y niñas cuenten con materiales e implementos para trabajar, aprender y desarrollarse. Es importante mencionar aquí que son los docentes los que deben realizar una constante revisión de sus implementos e informar regularmente a sus direcciones o encargados, ya que hoy día se cuenta con una importante subvención escolar en Chile, sobre todo la denominada Ley de subvención escolar preferencial (SEP) sería para solventar estas carencias. Se puede señalar con seguridad que hay variadas posibilidades para solventar materiales en cantidad y calidad, que pasan por procedimientos administrativos para su adquisición. Lamentablemente este proceso comienza con la proporción de la información de los profesionales a cargo de los talleres a los encargados correspondientes, procedimiento que en ocasiones no se hace o se desconoce.

La gran mayoría de los responsables de los talleres extraescolares corresponden a profesores de Educación Física, lo que da por deducción que las planificaciones, implementación y evaluaciones de los talleres están en manos de profesionales de la educación, ya que en Chile estos profesionales son formados en promedio nueve semestres. Esta formación incluye un acercamiento y fortalecimiento de la profesión a través de prácticas tempranas y constante reflexión para el desarrollo de experiencia y construcción identitaria docente que permite afrontar diversos desafíos formativos (Venegas \& Fuentealba, 2019), entre ellos los talleres. Por otra parte, una de las tareas de todas las instituciones educativas en Chile es aumentar la oferta de práctica de actividad física deportiva o de otra índole. La encuesta Nacional de Actividad física y Deporte de Chile (MINDEP, 2019) señala que el $78 \%$ de la población escolar no participa en otro taller o escuela donde realice deporte. Esta tarea debe quedar en manos de profesionales formados en el área, aunque no se puede desconocer que un profesional afín u otros responsables que aparecen mencionados en esta investigación como estudiantes universitarios, otros profesores, monitores o técnicos deportivos pueden tener habilidades y competencias para la enseñanza de los contenidos deportivos o de otra índole, así como alguna característica particular, formación especializada, experiencia como practicante de la disciplina u otra que los posicione en ese lugar. Por esta razón será importante que las instituciones educativas hagan el seguimiento del desarrollo y del aprendizaje de los participantes y responsables de los talleres. Sin duda, cobra especial importancia la presencia de profesores de Educación Física, ya sea los que tienen una carrera consolidada (más de 30 años), como los que recién la comienzan (más de 20 años), ya que según esta investigación son quienes se reparten la responsabilidad en los 48 talleres, y a quienes corresponde el gran desafío que se viene con los tiempos actuales de mantener a la población activa y saludable, diversificando los talleres deportivos a otros tipos de talleres activos, así como la diversificación a la población preescolar y escolar de primer ciclo que representa la menor participación y tiene la menor oferta.

Mención aparte merecen las mujeres responsables de los talleres extraescolares, las cuales solo alcanzan a representar un cuarto de estos. La Encuesta Nacional de Actividad física y Deporte de Chile (MINDEP, 2019) señala que el $52.7 \%$ de las mujeres entre 5 y 17 años se caracteriza como inactiva y el $32.5 \%$ como parcialmente activa. Si bien los profesionales a cargo no son los responsables de estas cifras, representan una tarea o un desafío pendiente que consiste en presentar propuestas para talleres extraescolares femeninos o ampliar la gama de talleres extraescolares que quieran practicar las damas, sin el afán de discriminar la danza o la expresión artística como una práctica puramente femenina, lo cual sigue siendo de gran interés para la población femenina y de escasa presencia en las instituciones educativas.

Como limitaciones del presente estudio se debe mencionar su carácter descriptivo y el reducido tamaño de la muestra, el cual no permite extrapolar los resultados obtenidos a toda la región de la Araucanía. En cuanto a las aplicaciones prácticas de este trabajo, sería recomendable que las instituciones educativas aumentaran el número de talleres recreativos, al ser estos los menos frecuentes y en el caso de aquellos que son de carácter formativo o competitivo sean diseñados de forma que puedan garantizar una secuencialidad de aprendizajes a través de los años escolares y un claro calendario de entrenamientos y competencias en los segundos.

Finalmente, es interesante resaltar que las mallas de las carreras de esta pedagogía se han concentrado preferentemente en el ámbito escolar curricular y los talleres extraescolares asoman como un campo profe- 
sional emergente que puede permitir profundizar y avanzar en el desarrollo de deportes individuales y colectivos, ya sean de los denominados tradicionales como de los emergentes, superando los fundamentos técnicos y tácticos básicos, para así comenzar con el desarrollo de talleres formativos deportivos al interior de los establecimientos educativos, principalmente de educación general básica. Según Bravo (2015) los talleres extraescolares enriquecen las propuestas curriculares de los establecimientos y generan condiciones que fortalecen las relaciones sociales beneficiando especialmente la integración a grupos de estudiantes con diversas capacidades y desarrollo de talentos. Por otro lado, basándose en las bases curriculares de educación física del MINEDUC, sería recomendable una secuenciación, profundización y explicitación de contenidos centrados en lo formativo y deportivo, pero complementando, profundizando, avanzando y formando año a año a los niños y niñas en determinados talleres deportivos. Es decir, una red de contenidos secuenciales como elemento común profesional sería la propuesta para la asignatura de educación física y salud y la formación deportiva en Chile, tal y como Díaz (2002) dijo: «las programaciones de los talleres extraescolares y las clases de Educación Física se constituyen en el eje central del avance de los aprendizajes de los niños y niñas españoles» (p. 90). De esta forma, los talleres extraescolares podrían ofrecer un salto cualitativo y cuantitativo en el proceso recreativo y deportivo escolar en Chile.

\section{Conclusión}

De acuerdo con los resultados de la presente investigación, se puede concluir que los talleres deportivos son los que predominan como actividades extraescolares y que mayoritariamente son dirigidos por profesionales de la Educación física. En el campo de la Educación Física estos resultados representan una oportunidad para enriquecer la propuesta curricular de los establecimientos ampliando la oferta tanto a deportes tradicionales como no tradicionales, emergentes, ancestrales, nacionales o extranjeros, individuales y colectivos, pero fomentando la participación femenina, la orientación recreativa en primera instancia y la continuidad en los talleres que requieran seguir con un proceso formativo coherente y organizado.

\section{Agradecimientos}

Los datos de este estudio fueron recogidos gracias al programa de Iniciación Científica 2020 realizado por la profesora de Pedagogía en Educación Física Javiera Morales Lagos y financiado por la Universidad Autónoma de Chile.

\section{Referencias}

Acosta, E. (2012). Iniciación y formación deportiva: una reflexión siempre oportuna. Revista UDCA:Actualidad \& divulgación cientifica. 15 (supl. Olimpismo). 57-65. Recuperado de: https:// repository.udca.edu.co/bitstream/11158/1997/1/893Texto\%20del\%20art\%c3\%adculo-3211-1-10-20180913.pdf

Arriagada, C. \& Calzadilla. O. (2019). Representaciones del profesoradodela educación básica araucana multigrado sobre las nuevas bases curriculares. RevistaEdusol. 19(68). 1-14. Recuperado de: http:// edusol.cug.co.cu/index.php/EduSol/article/view/1098.

Baeza, M., Morales. E. \& Suazo. A. (2017). Uso de las horas de libre disposición a 20 años de la implementación de la Jornada Escolar Completa [Tesis de pregrado. Universidad de Concepción]. Recuperado de: http://152.74.17.92/jspui/bitstream/11594/2467/3/ Baeza\%20-\%20Morales\%20-\%20Suazo.pdf

Bahamonde, C., Carmona, C., Albornoz, J., Hernández-Garcia, R., \& Torres-Luque, G. (2019). Efecto de un programa de actividades deportivas extraescolares enjóvenes chilenos. Retos, 35, 261-266. Recuperadode: https://doi.org/10.47197/retos.v0i35.62834

Beltrán,V., Sierra.A., Jiménez.A., González-Cutre. D., Martínez. C. \& Cervelló. E. (2017). Diferencias según género en el tiempo empleado por adolescentes en actividad sedentaria y actividad física en diferentes segmentos horarios del día. RETOS. NuevasTendencias en Educación Física. Deportey Recreación. 31. 3-7. Recuperado de: https: / / www.redalyc.org/articulo.oa?id=3457/345750049001

Bravo, J. (2015). Las prácticas que realizan los Asistentes de la Educación. durante los recreos en los patios de escuelas públicas básicas deValparaiso. y su relevancia en la gestión de la convivencia. [Tesis doctoral. Universitat Autonoma de Barcelona]. https://www.tdx.cat/bitstream/ handle/10803/383041/jsbg1de1.pdf?sequence $=1$ \&isAllowed $=\mathrm{y}$

Calero, D. (2016). Actividades extraescolares durante la adolescencia: Características que facilitan las experiencias óptimas. Psicoperspectivas. 15(2). 102-109. Recuperadode: https:/ /dx.doi.org/10.5027/psicoperspectivas-Vol15-Issue2-fulltext-856

Calvo, G. (2013). La formación de docentes para la inclusión educativa. Páginas de Educación. 6(1). 19-35. Recuperadode: http:/ /www.scielo.edu.uy/scielo.php?script $=$ sci_arttext\&pid $=\mathrm{S} 1688$ $74682013000100002 \& \operatorname{lng}=\mathrm{es} \& \operatorname{lng}=\mathrm{es}$

Carreño, J., Díaz,A., López, S., \& Martín, J. (2019). ¿Qué se investiga enformación docente en educación física y en recreación? (What is researched in teacher training in physical education and recreation?). Retos, 36, 3-8. Recuperado de: https://doi.org/ 10.47197/retos.v36i36.51456

Cruz, R. \& Castioni. R. (2019). Desafíos en la ampliación del tiempo escolar en dos contextos: Distrito Federal (Brasil) y Cataluña (España). Pro-Posições. 30. e20170095. Recuperado de: https: / / dx.doi.org/10.1590/1980-6248-2017-0095

Decreto $\mathrm{N}^{\circ} 290$. Diario oficial de la República de Chile. Santiago. Chile. 17 de septiembre de 1984.

Diaz, J. (2002). El currículum de la educación fisica en la reforma educativa. 
Editorial INDE. Barcelona. España.

Dirección de estudios sociológicos (DESUC) (2005). Evaluación de la Jornada Escolar Completa. Estudio de la Pontificia Universidad Católica de Chile Dirección de Estudios Sociológicos. Recuperado de: http: / / www.opech.cl/bibliografico/ Participacion_Cultura_Escolar/Informe_final_jec.pdf

García-Huidobro,J. \& Concha, C. (2009). Jornada escolar completa:la experiencia chilena. Recuperado de: https://docplayer.es/ 41236471-Jornada-escolar-completa-la-experiencia-chilena-documento-en-elaboracion-junio-de-2009-juan-eduardo-garciahuidobro-carlos-concha.html

Gurrola, M., Pimienta, J. \& Sil, L. (2015). La educación extraescolar en el Papalote. Museo del Niño. Instrumento para medir la calidad de las actividades. 4 (2). Recuperado de: file:///Users/imac/ Downloads/2.art\%C3\%ADculo\%20(1).pdf

Hermoso, Y. \& Chinchilla, J. (2010). La participación en actividades extraescolares de jóvenes y adolescentes y el nivel satisfacción en la ocupación de su tiempo libre. Revista Internacional de Psicología del DesarrolloylaEducación.2 (1).339-348.https:/ / www.redalyc.org/ articulo.oa2id $=3498 / 349832325034$

Instituto Nacional de Estadística (10 de marzo de 2021). Censos de población y vivienda.INE. https://www.ine.cl/estadisticas/sociales/cen$\mathrm{s}$ o s - d e - p o b l a c i o n - y -

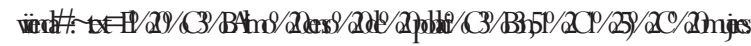
Instituto Nacional del Deporte. (2020). Bases de postulación al concurso público del fondo nacional para el fomento del deporte. convocatoria año 2021. Recuperado de: https: / /www.proyectosdeportivos.cl/ documentos_concursos/PDF/REX-2376.pdf

Manrique,A.\& Gallego,A. (2013). El material didáctico para la construcción de aprendizajes significativos. Revista Colombiana de Ciencias Sociales. 4(1). 101-108. Recuperado de: https:// dialnet.unirioja.es/descarga/articulo/5123813.pdf

Marambio Miranda, M., Núñez Fernández,T., Ramírez Guajardo, J., Ramírez Eyraud,P., Palma Leal, X., \& Rodríguez-Rodríguez., F. (2020). Relación entre la actividad física escolar y extraescolar en estudiantesde escuelas públicas chilenas (Relationshipbetweenschool and out-of-school physical activity in Chilean public school children). Retos, 37, 393-399. Recuperadode: https://doi.org/ 10.47197/retos.v37i37.72851

Martinic, S. (2015). El tiempo y el aprendizaje escolar la experiencia de la extensión de la jornada escolar en Chile. Revista Brasileira de Educação. 20(61). 479-499. Recuperado de: https://doi.org/ $10.1590 / \mathrm{S} 1413-24782015206110$

Medina Cascales, J., \& Prieto, M. J. (2019). Incidencia de la práctica de actividad física y deportiva como reguladora de la violencia escolar (Incidence of the practice of physical and sporting activities as a regulator of school violence). Retos, 35, 54-60. Recuperado de: https://doi.org/10.47197/retos.v0i35.64359

MINEDUC. (2001). El Reloj: sugerencias para la formulacion del proyecto pedagogico de Jornada Escolar Completa del establecimiento educacional: jornada escolar completa. MINEDUC.

MINDEP. (2019). Encuesta nacional de actividad física y deporte en menores de 5 a 17 años. Recuperado de: https: / /ligup-v2.s3-saeast-1.amazonaws.com/sigi/media/Resumen-Encuesta-deh\%C3\%A1bitos-2019.pdf

MIME.MINEDUC. (2020). Másinformación mejor educación. Recuperadode: https://www.mime.mineduc.cl/mvc/mime/portada
MINDEP.(18 de mayo de 2020). Ministerio del Deporte. Mindep realiza la primera Encuesta Nacional de Actividad Física y Deportes en Sudamérica en niños y jóvenes. Recuperado de: https:// www.mindep.cl/actividades/noticias/1559

Millalen,A. (2008). LostalleresJEC una estrategia para mejorar los aprendizajes de los estudiantes en las escuelas rurales de Laja [Tesis de Magister. Universidad del BíO-BÍO]. http://repobib.ubiobio.cl/jspui/ bitstream/123456789/2104/1/Millalen_Suazo_Alejandra.pdf

Nogueira, H. (2008). Elderechoala educación y sus regulaciones básicas en el derecho constitucional chileno e internacional de los derechos humanos. Iuset Praxis. 14(2). 209-269. Recuperado de: https: / / dx.doi.org/10.4067/S0718-00122008000200007

Ley 19532. Diario oficial de la República de Chile. Santiago. Chile. 17 de noviembre de 1997.

Ley 20248. Diario oficial de la República de Chile. Santiago. 01 de febrero de 2018.

Ley 20370. Diario oficial de la República de Chile. Santiago. Chile. 12 de septiembre de 2009.

Parra,A., Oliva.A.\&Antolín. L. (2009). Los programas extraescolares como recurso para formentar el desarrollo positivo adolescente. Papeles del Psicólogo.30(3). 3-13. Recuperadode:https:/ /idus.us.es/bitstream/handle/11441/16945/file_1.pdf

Ponce de León-Elizondo,A., Sanz-Arazuri,E., Sáenz de Jubera-Ocón, M., \& Alonso-Ruiz, R. (2019). La jornada lectiva a debate para (re)convertir los centros educativos en espacios para el ocio. Revista DeInvestigación Educativa. 37(2). 395-411. Recuperadode: https:/ /doi.org/10.6018/rie.37.2.322751

Sanabria Navarro, J., Silveira Pérez,Y., Guillen Pereira, L., \& Pérez Bravo, D. (2019). Emprendimientos deportivos: Caso Subregión Sabana, Departamento de Sucre, Colombia (Sport entrepreneurship: Sub-region Sabana, Department of Sucre, Colombia). Retos, 35, 140-146. Recuperado de: https://doi.org/ 10.47197/retos.v0i35.64124

Sandoval, P., \& García, I. (2014). Cultura deportiva en Chile: desarrollo histórico, institucionalidad actual e implicancias para la política pública. Polis(Santiago), 13(39), 441-462. Recuperadode:https:/ /dx.doi.org/10.4067/S0718-65682014000300020

Salazar, B. (2020). El fútbol formativo como herramienta motriz de aprendizaje (Doctoral dissertation, Corporación Universitaria MinutodeDios). Recuperadode:https: / / repository.uniminuto.edu/ handle/10656/10511

SPSS. (27 de enero de 2021). Universitat de Barcelona. Estadística. Prueba Chi-Cuadrado. Recuperadode:http://www.ub.edu/aplica_infor/ spss/cap5-2.htm

Trujillo, L. (2014). La importancia de los espacios escolares en la enseñanzaaprendizaje de los alumnos [Tesis de pregrado. Universidad de Málaga]. Recuperado de: https://riuma.uma.es/xmlui/bitstream/ $\mathrm{h}$ a $\mathrm{n} \mathrm{d} \mathrm{l} \mathrm{e} \mathrm{/} 100630 / 9006 \%$ / TrujilloBenitez_TFG_Primaria.pdf?sequence $=1$

Vanegas, C. \& Fuentealba,A. (2019). Identidad profesional docente. reflexión y práctica pedagógica: Consideraciones claves para la formación de profesores. Perspectiva Educacional. 58(1). 115-138. Recuperadode: https://dx.doi.org/10.4151/07189729-vol.58-iss.1art.780 